\title{
Guidelines
}

Skin

Appendage

Disorders

\section{Look at Your Hair the Way You Look at Your Face: Concept of Total Facial Skin and Hair Care}

\author{
Murilo Calvo Peretti ${ }^{a}$ Natalia Caballero Uribe ${ }^{b}$ Antonia Régnier ${ }^{b}$ \\ Ralph M. Trüeb ${ }^{b}$ \\ aDepartment of Dermatology, Clinics Hospital (Hospital de Clínicas), Federal University of Parana (HC-UFPR), \\ Curitiba-Paraná, Brazil; ${ }^{b}$ Center for Dermatology and Hair Diseases Professor Trüeb, Zurich-Wallisellen, Switzerland
}

\section{Keywords}

Skin and hair aging · Primary, secondary, and tertiary prevention - Total facial skin and hair care concept

\begin{abstract}
With today's increasing life expectation and quality of life, the desire to look young and beautiful plays a bigger role than ever. Aging of the hair is particularly visible. And yet, cosmetic dermatology has paid comparatively little attention to the care of the aging hair. The professional market for cosmetic interventions into the face has preceded those into the hair for a number of reasons. First, the market for facial rejuvenation has been dominated by plastic surgeons, until the fillers and botulinum toxin were introduced. Second, investigative dermatology found a profound interest in the study of skin aging, particularly as it relates to ultraviolet exposure, and exposed mechanisms at the level of the DNA and repair mechanisms, opening venues for effective preventive measures and pharmacological treatments of agingrelated conditions of the skin. Finally, at the level of health professionals, the care for the skin has been overrated in relation to the hair, mostly due to economic reasons. Nevertheless, the hair represents an integral part of the face and our appearance. Moreover, the discovery of pharmacologi-
\end{abstract}

cal targets and the development of safe and effective drugs for treatment of hair loss indicate strategies of the drug industry for maintenance of hair growth and quantity, while the hair care industry has become capable of delivering active compounds directed toward meeting the consumer demand for maintenance of hair cosmesis and quality. To offer patients the best outcomes toward a more youthful look, we therefore should look at the whole face including the condition of the hair for a more comprehensive approach toward a total facial skin and hair care concept.

(c) 2020 S. Karger AG, Basel

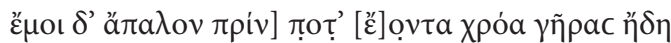

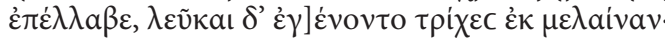

(My skin was once supple and smooth, but now it is withered by age; my hair had been lustrous and black, but now it is faded and gray.) Sappho (c. 630 - c. 570 BC)

Hair care, color, and style play an important role in people's overall physical appearance and self-perception. Hair defines an individual's gender, sexual attitude, and social status. Eventually, aging of the hair is particularly visible. And yet, cosmetic dermatology has paid comparatively little attention to the care of the aging hair, while cosmetic dermatologic procedures for the face have

\section{KARGER}

(c) 2020 S. Karger AG, Basel

karger@karger.com

www.karger.com/sad
Ralph M. Trüeb, MD

Center for Dermatology and Hair Diseases Professor Trüeb Bahnhofplatz 1A

$\mathrm{CH}-8304$ Wallisellen (Switzerland)

r.trueeb@derma-haarcenter.ch 
evolved to a booming industry. Nevertheless, the discovery of pharmacological targets and the development of safe and effective drugs for treatment of hair loss indicate strategies of the drug industry for maintenance of hair growth and quantity, while the hair care industry has become capable of delivering active compounds directed toward meeting the consumer demand for maintenance of hair cosmesis and quality [1].

It is the aim of this paper to introduce the cosmetic dermatologic interventions for aging hair as an integral part of a total hair and facial skin care concept.

\section{Demographics of Aging}

Worldwide the demographics of aging have changed. For most of history, populations have been young and lives short. Population aging is mainly due to 2 developments: First, people today are living longer than before; second, there is a decline in the birth rate, resulting in smaller younger generations relative to older generations, raising the average age of the population [2].

\section{What Is Beauty?}

Beauty is an abstract concept and has been an object of interest and discussion among both philosophers and evolutionary scientists. Beauty is understood as an individual's subjective appraisal of attractiveness that is influenced by cultural standards. Sociocultural images of beauty are best reflected in a variety of popular beauty icons. However, despite some unique cultural variabilities in esthetic judgments, evidence has shown that similar patterns emerge across different societies [3]. Indeed, a set of convincing studies confirm that our perception of attractiveness predate cultural influences. In his seminal "The Descent of Man, and Selection in Relation to Sex" [4], Charles Darwin (1809-1882) reflected on the physical characteristics that seemed to act as open lures to predators ant therefore interfere with survival. For example, how could the brilliant plumage of peacocks have evolved? Darwin's answer was sexual selection, that is, that certain characteristic evolved because of reproductive advantage rather than survival advantage. The evolutionary argument hypothesizes that physical signs of youth and health, such as full lips, smooth skin, clear eyes, lustrous hair, good muscle tone, animated facials expression, and high energy level, are at the top of every culture's beauty list, simply because they are the most reliable physical signals for fertility. Contemporary research has attempted to identify the physical features that account for the attractiveness of an individual and has recognized several factors: facial and body symmetry [5], averageness of appearance (koinophilia) [6], body-size ratios [7], and youthfulness [8].

Youthfulness in particular marks an extended period of reproductive potential. Looking young may be more important than actually being young, and altering facial features in the direction of youth results in higher ratings of attractiveness [9]. In contrast to the substantial literature on the psychology of physical appearance and attractiveness, relatively little has been published on the impact of the condition of the hair. Nevertheless, respective research into the subject has corroborated that loss of hair diminishes perceived youthfulness and attractiveness [10].

\section{Definition of Procedures to Divert the Signs of Aging}

The antiaging movement is a societal movement that has committed itself to prevent, delay, or reverse the effects of aging, at least at the level of recognition. While a significant portion of the attention of the movement originally has been on the possibilities for life extension, today there is a more important interest in techniques to ameliorate the effects of aging rather than delay or defeat them. Central to antiaging medicine have originally been the administration of hormones, such as menopausal hormone drugs, and human growth hormone, and of supplements, while studies into their efficacy have eventually revealed that none are effective in extending life [11], while some may even carry health risks. Therefore, the current market has changed its focus onto lifestyle treatments (better aging) to include: weight reducing agents, agents to treat erectile dysfunction, mood-modifying agents, cosmetic surgery, minimal invasive cosmetic dermatologic procedures, and hair growth-promoting agents.

On the occasion of a symposium on lifestyle drugs and aesthetic medicine, the Dorian Gray syndrome (named after Oscar Wildes Gothic horror novel "The Picture of Dorian Gray") was proposed as a contemporary cultural phenomenon, characterized by an extreme pride in one's own appearance accompanied by difficulties coping with the aging process. By definition, individuals with the condition are users of cosmetic medical procedures and products in an attempt to preserve their youth. An estimated 3\% of the Western population displays features of
Calvo Peretti/Caballero Uribe/Régnier/ Trüeb 
the condition [12]. And yet, in its original meaning in Koine Greek the word for beautiful was ẃpaıo, an adjective etymologically coming from the word $\omega \rho \alpha$, meaning "hour". Beauty was thus associated with "being of one's hour". Consequently, a ripe fruit (of its time) was considered beautiful, whereas a young individual trying to appear older, or an old individual trying to appear younger would not be considered beautiful. Examples of disastrous results of excessive plastic surgery and cosmetic dermatologic procedures can be found under www. oddee.com/item_96587.aspx.

\section{Cosmetic Interventions for Facial Skin and Hair}

The professional market for cosmetic interventions into the face has preceded those into the hair for a number of reasons.

First, the market for facial rejuvenation has been dominated by the plastic surgeons, until the fillers and botulinum toxin were introduced.

Second, investigative dermatology found a profound interest in the study of skin aging, particularly as it relates to ultraviolet exposure, and exposed mechanisms at the level of the DNA and repair mechanisms, opening venues for effective preventive measures of aging and pharmacological treatments of aging-related conditions of the skin.

Finally, at the level of the health professional, the condition of the skin has been overrated in relation to the hair, mostly due to economic reasons. And yet, the hair represents an integral part of the face and our appearance, with culture-bound, sex- and age-dependent grooming habits. At all times, hair coloring, hair pieces, and wigs have represented ways of coping with precocious loss of hair and color at the level of the consumer. Eventually, hair transplantation has emerged, while a more profound investigation into to basics of hair aging, its prevention, and treatment have lagged the advances in skin antiaging [13].

\section{Facial Skin Aging}

Facial skin aging is caused by intrinsic and extrinsic mechanisms. Intrinsic skin aging represents the normal course of aging for all tissues, whereas extrinsic aging is mainly caused by exposure to UV radiation, pollution, and cigarette smoking that is superimposed on intrinsic or chronological skin aging. Exposed areas of the body such as the face are, therefore, subjected to both types of skin aging [14].

Concept of Total Facial Skin and Hair

Care
The first signs of the aging face are usually visible by the third decade of life, when eyebrows start to descend as a result of increased skin laxity, gravitational force, and repeated contractions of the facial muscles of the periorbital region [15]. Progression of the aging process in the following decades produces additional changes in facial soft tissues and bony landmarks, leading to alterations in facial proportions $[15,16]$.

Age-dependent reduction of sexual hormone levels result in skin dryness, wrinkling, epidermal thinning, loss of collagen, and increased skin laxity. The skin becomes more fragile and its protective functions are reduced [14]. Histologic features of intrinsic aging include reduction in epidermal thickness by $10-50 \%$ with atrophy of the stratum spinosum due to reduced basal keratinocyte proliferation and increased size heterogeneity $[17,18]$. The dermis becomes atrophic and hypocellular, with diminished microvasculature. Dermal collagen, elastin, and glycosaminoglycans are altered. The subcutaneous tissue is reduced in some areas, like the face, and finally, the number of both sebaceous and sweat glands is reduced $[19,20]$.

\section{Procedures to Prevent, Delay, or Reverse the Signs of Facial Skin Aging}

Facial skin is particularly exposed to the influence of extrinsic factors, which carries the risk of overexposure and consequent prematurely aged skin. The human face reflects the first signs of aging, including structural, functional, and esthetic changes, accompanied with various benign, premalignant, and malignant pathologic conditions. Since a substantial proportion of skin changes are related to extrinsic factors they are preventable [21].

The skin is normally equipped with protective antioxidant systems, including chemical and enzymatic antioxidants. Chemical antioxidants can be water soluble (vitamin C, glutathione, lipoic acid, uric acid), or lipid soluble (vitamin $\mathrm{E}$ and other tocopherol forms, ubiquinone, carotenoids). Enzymatic antioxidants include superoxide dismutase, catalase, glutathione peroxidase, and glutathione transferase. These antioxidants protect the skin from reactive oxidant species and free radicals; however, with age and environmental stress, such as UV and ozone exposure, the capacity of these antioxidant systems is diminished, making the skin more vulnerable to damage $[22,23]$. 


\section{Primary, Secondary, and Tertiary Prevention}

Over time, the definition of prevention has expanded so that its meaning in the context of health services has evolved. Prevention, in a narrow sense, means averting the development of a pathological condition. In a broader sense, it includes all measures that limit the progression of the pathology at any stage of its course. Originally, a distinction was made between interventions that avert the occurrence of a pathologic condition (primary prevention) and interventions that halt or slow the progression of that condition or its sequelae at any point after its inception (secondary prevention). Eventually, the distinctions between types of prevention had expanded to include primary prevention to promote health prior to the development of pathology; secondary prevention to detect pathology in early stages and to reverse, arrest, or delay progression, and tertiary prevention to improve function, minimize impact, and delay complications.

As risk factors are increasingly considered to be the equivalent of pathology for purposes of intervention, the concept of prevention is losing all practical meaning [24].

The mainstay of protection of the skin from premature aging is adequate photoprotection and abstinence from smoking. Photoprotection is achieved by sun avoidance, sun-protective clothing, and sunscreens [21]. A variety of antioxidants can be included in topical products to boost the endogenous antioxidant systems within the skin. These include the common vitamins already found in the skin, activators of antioxidant enzyme systems present within the skin, or herbal actives with strong antioxidant properties [25].

For the most natural and harmonious rejuvenation of the face, all changes that result from the aging process should be corrected. Aging is a result of the interplay of changes occurring in all facial anatomical layers: skeleton, ligaments, muscles, adipose tissue, and skin. To target these, multilayer, combined intervention is required to relax, volumize, resurface, and re-drape facial skin. No single technology, filler, or neuromodulator can achieve all the results desired in treating the aging face. The question is when and how to combine different esthetic interventions safely and effectively for the face, hands, neck, and décolletage. Optimal outcomes are dependent upon choosing the appropriate technique and ensuring that it is used correctly. A profound understanding of product characteristics, anatomy, and the physiology of aging is essential to know when, where, and how to use the different modalities to provide facial harmony [14].
Skin glow, texture, and pigmentation have an equally significant impact on attractiveness. Healthy skin can be retained with the use of topical skincare products, beyond daily UV protection and topical antioxidants, to include moisturizers, topical retinoids, hyaluronic acid fragments, bioactive peptides, and DNA repair enzymes, and some device-based rejuvenating procedures (intense pulsed light, radiofrequency) for prevention or early treatment of irregular pigmentation, superficial skin laxity, and/or fine wrinkles [14].

\section{Hair Aging}

Aging of hair affects hair color, hair production, and structural properties of the hair fiber with its consequence for manageability and overall appearance of the hair. While most dermatological literature on age-related hair changes has focused on hair loss, it is equally important that the hair fibers that emerge from the scalp exhibit significant age-related changes that have an equal impact on the overall cosmetic properties of the hair [26]. Depending on the individual's original hair color, quantity, quality, and hair care habits, there is a great variability in the age of onset of first signs of hair aging.

Age-related deterioration of cosmetic properties of hair is related to changes in hair pigmentations (graying), diameter, curvatures, structural properties (stretching, bending, torsional rigidity), lipid composition, and the interdependence of these changes. It is these changes that are ultimately experienced by subjects that have retained their hair as they age [26].

Meanwhile, basic scientists interested in the biology of hair growth and pigmentation have exposed the hair follicle as a highly accessible model with unique opportunities for the study of age-related effects [27]. The hair follicle's complex multicell type interaction system involving epithelium, mesenchyme, and neuroectoderm, and its unique cyclical activity of growth, regression, rest and regrowth provide the investigator with a range of stem, differentiating, mitotic and postmitotic terminally differentiated cells, including cells with variable susceptibility to apoptosis, for study. As such, human hair follicle melanocytes have even been used as a proxy cell type in neurodegeneration research [28].

Finally, clinical dermatologists involved in the care for hair have discovered dermoscopy to be useful in their daily clinical practice. Originally developed for looking at the skin to distinguish malignant pigmented skin lesions, the use of the dermoscope has been extended to evaluate oth- 
er dermatological conditions, and eventually to look at the hair and scalp, where it is called trichoscopy [29]. Expert opinion suggests that trichoscopy improves diagnostic capability beyond simple clinical inspection. Ultimately, trichoscopy reassures patients complaining of hair loss that they are receiving a thorough examination, since they often feel that they are not properly examined. The method allows viewing at high magnifications using a simple hand-held dermoscope, and is combined with photography and digital imaging for clinical documentation purposes in the course of treatments for hair loss [30].

In pattern hair loss, major advances were achieved in the understanding of peculiarities of the androgen metabolism involved [31]. Since many extrinsic hair growthmodulatory factors, such as androgens [32], apparently operate at least in part via the dermal papilla, research is focused on identifying androgen-regulated factors deriving from dermal papilla cells. Of the factors that have been suggested to play a role in hair growth, insulin-like growth factor has been reported as altered in vitro by androgens [33], underlining its role for hair growth [34]. Nevertheless, the limited success rate of treatment of pattern hair loss with modulators of androgen metabolism, such as the 5alpha-reductase inhibitors, means that further pathogenic pathways must be taken into account with oxidative stress and follicular microinflammation and fibrosis currently being the focus of current investigations.

Naito et al. [35] analyzed the effect of the lipid peroxides on hair follicles and observed that the topical application of linolein hydroperoxides, one of the lipid peroxides, lead to the early onset of the catagen phase in murine hair cycles. Furthermore, they found that lipid peroxides induced apoptosis of hair follicle cells. They also induced apoptosis in human epidermal keratinocytes by upregulating apoptosis-related genes. These results indicate that lipid peroxides, which can cause free radicals, induce the apoptosis of hair follicle cells, and this is followed by early onset of the catagen phase.

Bahta et al. [36] cultured dermal hair papilla cells (DPC) from balding and nonbalding scalp and originally demonstrated that balding DPCs grow slower in vitro than nonbalding DPCs. Loss of proliferative capacity of balding DPCs was associated with changes in cell morphology, expression of senescence-associated beta-galactosidase, decreased expression of proliferating cell nuclear antigen and Bmi-1, upregulation of p16(INK4a)/ $\mathrm{pRb}$, and nuclear expression of markers of oxidative stress and DNA damage including heat shock pro-

Concept of Total Facial Skin and Hair Care tein-27, super oxide dismutase catalase, ataxia-telangiectasia-mutated kinase (ATM), and ataxia-telangiectasia-mutated kinase- and Rad3-related protein. The finding of premature senescence of balding DPC in vitro in association with expression of p16(INK4a)/pRB suggests that balding DPCs are particularly sensitive to environmental stress.

In view of this role of oxidative stress, the question arises whether the pattern hair loss should not be redefined from a genetically determined, androgen-induced, age-dependent, progressive hair loss with sex-dependent differences in incidence, pattern and severity to: a genetically determined, organ-specific (hair follicle) accelerated aging process with increased sensitivity to internal and external factors of hormonal, environmental, inflammatory, vascular, and dietary origin, with its implications for treatment strategies beyond minoxidil and the 5alpha-reductase inhibitors, to include nutritional, anti-inflammatory, anti-apoptotic, low level laser, microneedling, mesotherapy, and cell-based therapies [37].

Finally, the medical focus has traditionally been either on hair loss or on the condition of the scalp in terms of specific dermatologic conditions, while in fact, the proximate structural arrangement of the scalp and hair leads to an interdependent relationship between the two. The protective benefits of the hair to the scalp, such as ultraviolet radiation screening, moisture retention, and mechanical shielding, are obvious, while the role of the scalp as an incubatory environment for the pre-emergent hair fiber is often overlooked [38]. Nonetheless, there is a wealth of observational data on specific dermatological conditions of the scalp, such as dandruff and seborrheic dermatitis, atopic dermatitis, and psoriasis, providing the evidence for the role of the scalp condition in supporting the production of healthy hair. Again, oxidative stress is prevalent in all of these skin conditions, just as in normal skin aging, and related to the specific microbiome of the scalp and to the environmental exposures [39].

\section{Procedures to Prevent, Delay, or Reverse the Signs of Hair Aging}

As in the rest of antiaging medicine, the aim of hair anti-aging is to delay, reduce, or reverse the effects of aging on hair. According to the complex nature of the aging process, the treatment for lifetime scalp and hair health has to be holistic to include the multitude of contributing factors in a polyhedral and patient-specific 


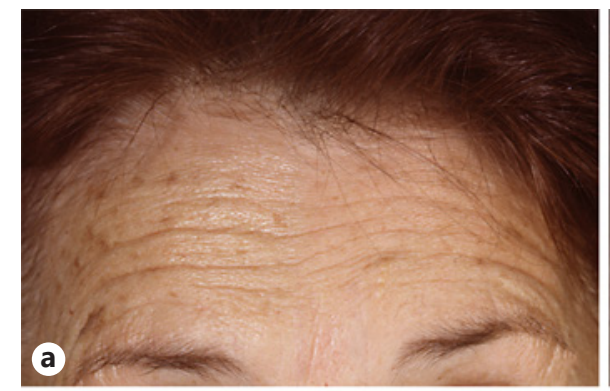

Fig. 1. Skin anti-aging of the upper and of the lower face: treatment of forehead wrinkles with botulinum toxin $(\mathbf{a}, \mathbf{b})$, before (a) and after (b) treatment, and treatment of perioral wrinkles $(\mathbf{c}, \mathbf{d})$ with injectable hyaluronic acid, before (c) and after (d) treatment (courtesy of Monika Hess, MD, Zürich, Switzerland; courtesy of Monika Hess, MD, Zürich, Switzerland).

Fig. 2. Skin photorejuvenation with intense pulsed light: lentigines (a, b), facial teleangiectasia (c, d), neck erythrosis (e, f), before $(\mathbf{a}, \mathbf{c}, \mathbf{e})$, and after (b, d, f) treatment (courtesy of Wolfgang Thürlimann, MD, Zürich, Switzerland).

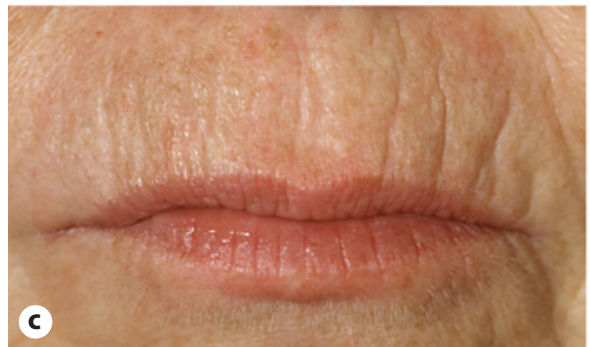

b
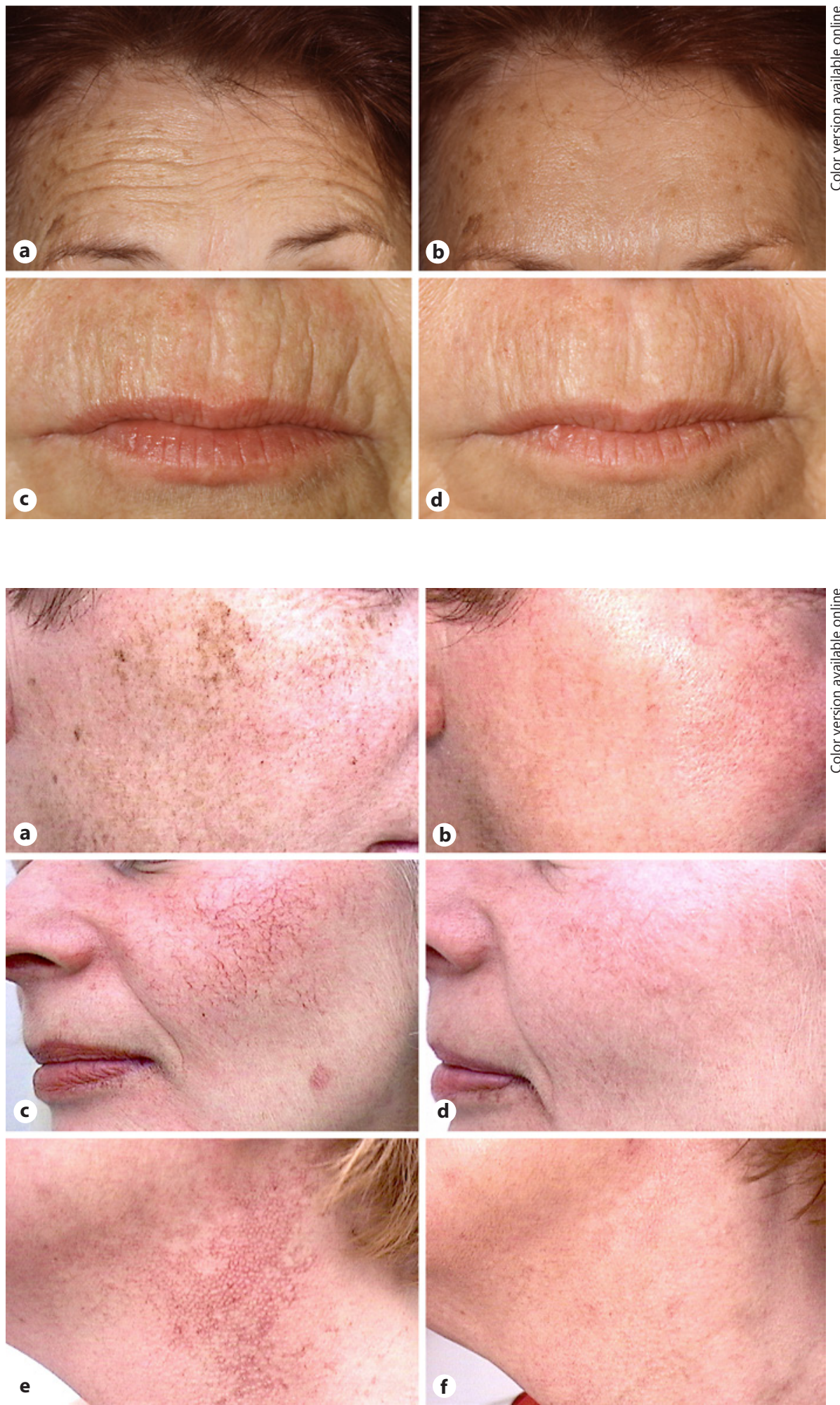

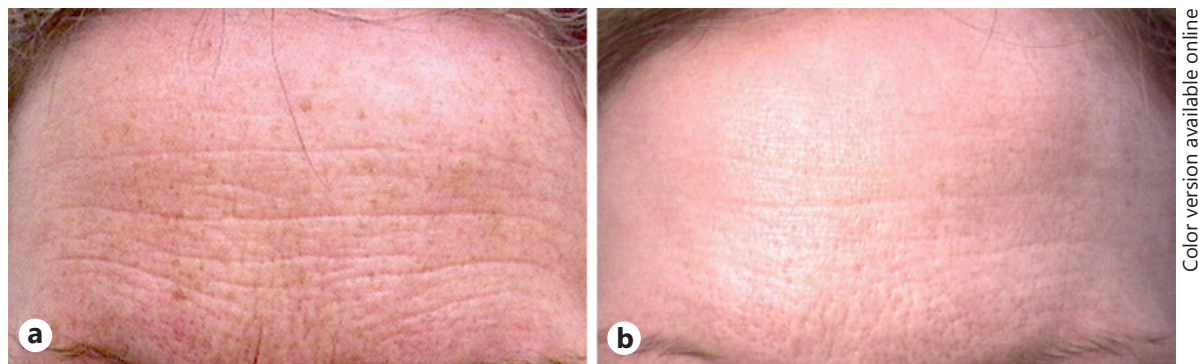

Fig. 3. Skin antiaging (total face): total face resurfacing with $\mathrm{CO}_{2}$ laser, before $(\mathbf{a}, \mathbf{c})$ and after (b, d) treatment (courtesy of Wolfgang Thürlimann, MD, Zürich, Switzerland).
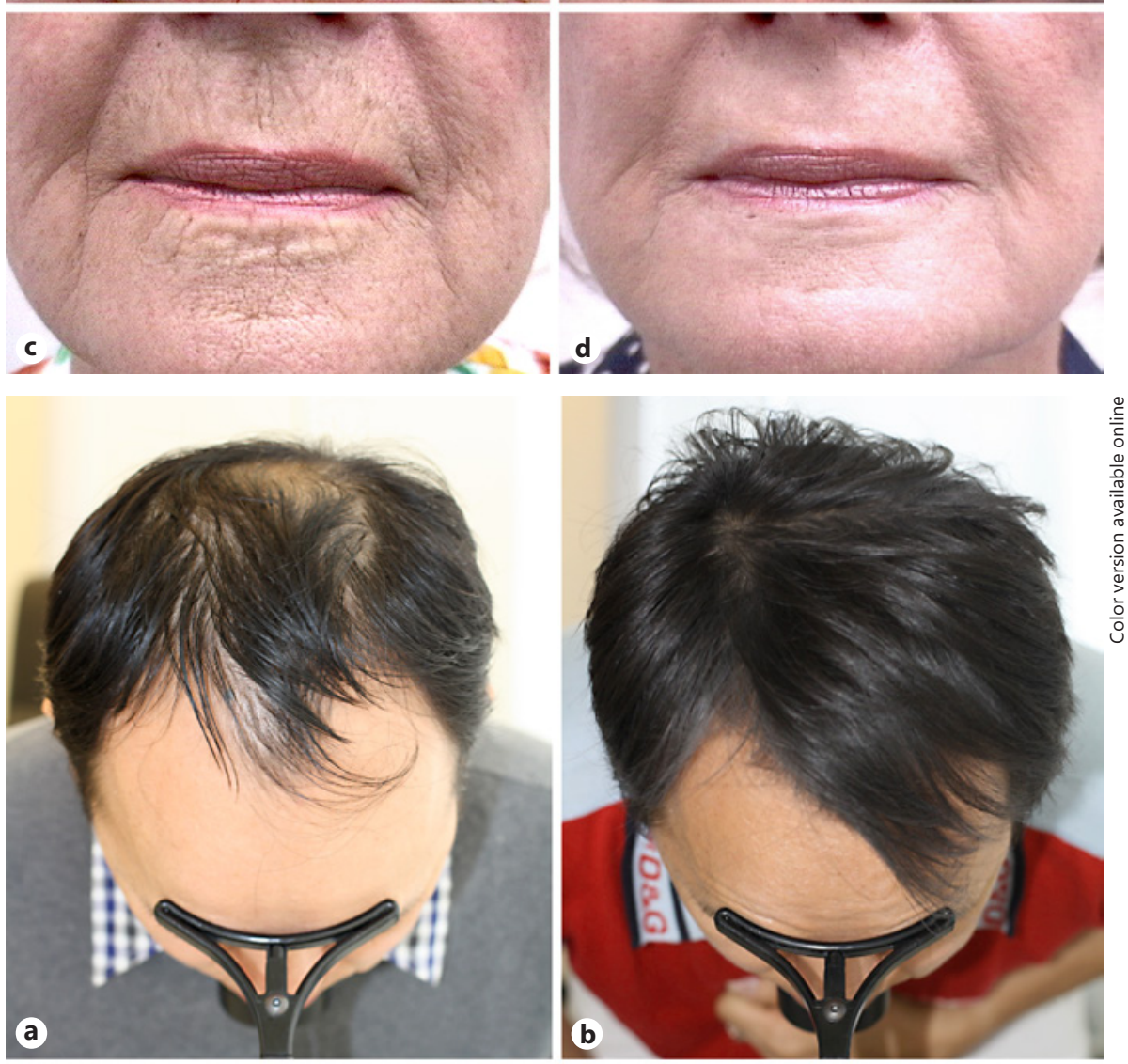

Fig. 4. Hair antiaging: treatment of male (a, b) and female $(\mathbf{c}, \mathbf{d})$ pattern hair loss with combination of $1 \mathrm{mg}$ orale finasteride and $5 \%$ topical minoxidil b.i.d. (a, b), before (a) and after (b) treatment, and 5\% topical minoxidil b.i.d. (c, d) resp., before (c) and after (d) treatment (Center for Dermatology and Hair Disease Professor Trüeb).
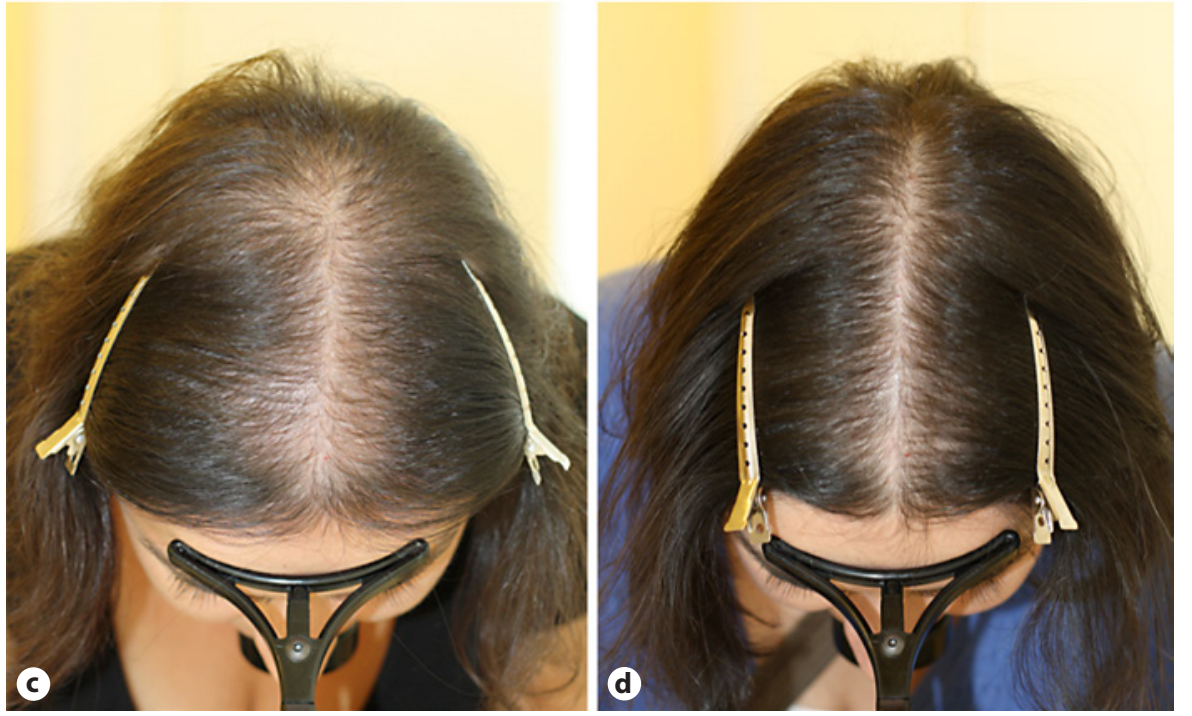
Fig. 5. a, b "Man can have only a certain number of teeth, hair and ideas; there comes a time when he necessarily loses his teeth, hair and ideas," Voltaire (16941778 ) at 82 , without and with perruque, by J.A. Houdon (photograph taken by R.M.T. at National Gallery of Art, Washington, DC, USA).
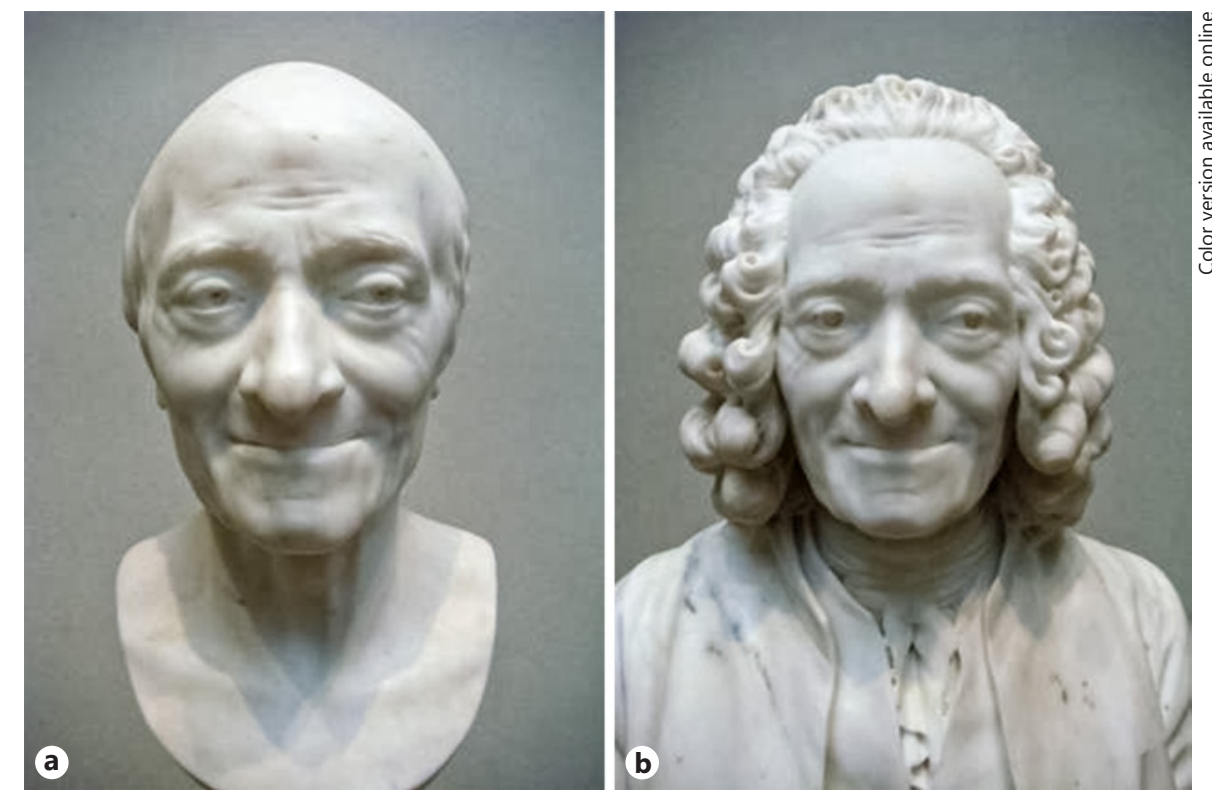

manner. It comprises both medical treatments and hair cosmetics.

Medical treatments include: specific pharmacological treatments of patterned hair loss, such as topical minoxidil and 5alpha-reductase inhibition, pharmacological treatments of specific dermatological conditions of the scalp, such as dandruff and seborrheic dermatitis, and management of age-related general health problems affecting the condition of the hair: nutritional, endocrine, psychological, drug-related, substance abuse (including smoking [40]), and multimorbidity.

Since there is a decrease in 5alpha-reductase activity in senescent alopecia [41], efficacy of the 5alpha-reductase inhibitors decreases in men after the age of 60 years. Given that minoxidil exerts its effect independent of androgen metabolism, and fibrosis is not a feature of senescent alopecia, minoxidil works well in these men who have retained some hair. Moreover, minoxidil is effective in women irrespective of estrogen levels, and therefore retains its efficacy in women after menopause, and in women undergoing endocrine therapy for breast cancer [42].

The value of the special ingredients shampoos cannot be underscored as additional elements to enhance the action of the pharmaceutical topical or oral treatments by cleansing the scalp and contributing to a healthy scalp microbiome. The use of appropriate surfactants combined with ingredients, such as zinc pyrithione, ciclopirox, salicylic acid [43-46], copper chelating agents, such as
$N, N$-ethylene diamine disuccinic acid [47], and others, contribute to maintain Malassezia spp. and oxidative stress under control.

Cosmetic treatments include: appropriate hair grooming habits, appropriate shampoo selection, conditioner use, hair coloring, hair styling aids, and hair and scalp photoprotection. The efficacy of hair growth-promoting agents or antiaging substances in shampoo form is questionable given their dilution with water and short contact time, unless they can be absorbed in effective quantities. Nevertheless, cosmetic products represent a key integral part of management of aging hair, since they may significantly improve the condition of the hair fiber with more immediate effects, while pharmacologic agents take their time. Specifically, a leave-on technology combination of caffeine, niacinamide, panthenol, dimethicone, and an acrylate polymer has been demonstrated to affect the diameter and behavior of individual terminal scalp hair fibers as an approach to counteract decreasing fiber diameters. Beyond the diameter increase, the caffeine, niacinamide, panthenol, dimethicone, and an acrylate polymer-thickened fibers also demonstrated increased suppleness/pliability (decreased shear modulus) and better ability to withstand force without breaking (increased break stress). Although cosmetic treatments will not reverse the process of hair aging, such technologies help mitigate the effects of age-related thinning of hair [48]. 


\section{Summary and Conclusions: Concept of Total Facial Skin and Hair Care}

To offer patients the best outcomes toward a more youthful and healthy look, we should think beyond partial interventions, look at the whole face at its different tissue depths, and at the condition of the hair to determine the appropriate approach to a comprehensive treatment with emphasis on recognizing that more than one procedure will be required to address all issues in question: injectables, topical skin care, energy-based therapies, hair growth-promoting agents, nutritionals, and sophisticated hair care products should be combined for optimal results within a total facial skin and hair care concept (Fig. 1-5). Ultimately, postgraduate training into cosmetic dermatology should integrate both, the facial skin and the hair, as they relate to age, the environment, the general health, and nutritional status, in their syllabus [49].

\section{Statement of Ethics}

The authors have no ethical conflicts to disclose.

\section{Disclosure Statement}

This paper represents an integral part of M.C.P. traineeship in trichiatry at the Center for Dermatology and Hair Diseases Professor Trüeb. R.M.T. is active member of the Healthy Hair and Scalp Alliance and in this function provides consulting activity for Procter and Gamble.

\section{References}

1 Trüeb RM; Swiss Trichology Study Group. The value of hair cosmetics and pharmaceuticals. Dermatology. 2001;202(4):275-82.

2 Weil DN. The Economics of Population Aging. In: Rosenzweig MR, Oded S, editors. Handbook of Population and Family Economics. New York: Elsevier; 1997. p. 9671014.

3 Sarwer DB, Grossbart TA, Didie ER. Beauty and society. Semin Cutan Med Surg. 2003 Jun; 22(2):79-92.

4 Darwin C. The Descent of Man, and Selection in Relation to Sex. London, United Kingdom: John Murray (pulisher); 1871.

5 Baudouin JY, Tiberghien G. Symmetry, averageness, and feature size in the facial attractiveness of women. Acta Psychol (Amst). 2004 Nov; 117(3):313-32.

6 Valentine T, Darling S, Donnelly M. Why are average faces attractive? The effect of view and averageness on the attractiveness of female faces. Psychon Bull Rev. 2004 Jun;11(3): 482-7.

7 Singh D. Waist-to-hip ratio and judgment of attractiveness and healthiness of female figures by male and female physicians. Int J Obes Relat Metab Disord. 1994 Nov; 18(11):731-7.

8 Marshall C, Lengyel C, Utioh A. Body dissatisfaction among middle-aged and older women. Can J Diet Pract Res. 2012;73(2):e241-7.

9 Ehlinger-Martin A, Cohen-Letessier A, Taïeb M, Azoulay E, du Crest D. Women's attitudes to beauty, aging, and the place of cosmetic procedures: insights from the QUEST Observatory. J Cosmet Dermatol. 2016 Mar;15(1): 89-94.

10 Cash TF. Losing hair, losing points? The effects of male pattern baldness on social impression formation. J Appl Soc Psychol. 1990; 20(2):154-67.
11 Kamel NS, Gammack J, Cepeda O, Flaherty $\mathrm{JH}$. Antioxidants and hormones as antiaging therapies: high hopes, disappointing results. Cleve Clin J Med. 2006 Dec;73(12):1049-56.

12 Brosig B, Kupfer J, Niemeier V, Gieler U. The "Dorian Gray Syndrome": psychodynamic need for hair growth restorers and other "fountains of youth". Int J Clin Pharmacol Ther. 2001 Jul;39(7):279-83.

13 Trüeb RM, Rezende HD, Dias MF. A comment on the science of hair aging. Int J Trichology. 2018 Nov-Dec;10(6):245-54.

14 Fabi S, Pavicic T, Braz A, Green JB, Seo K, van Loghem JA. Combined aesthetic interventions for prevention of facial ageing, and restoration and beautification of face and body. Clin Cosmet Investig Dermatol. 2017 Oct;10: 423-9.

15 Sadick NS, Karcher C, Palmisano L. Cosmetic dermatology of the aging face. Clin Dermatol. 2009;27 Suppl 3:S3-12.

16 Ramos-e-Silva M, Celem LR, Ramos-e-Silva S, Fucci-da-Costa AP. Anti-aging cosmetics: facts and controversies. Clin Dermatol. 2013 Nov-Dec;31(6):750-8.

17 Kohl E, Steinbauer J, Landthaler M, Szeimies RM. Skin ageing. J Eur Acad Dermatol Venereol. 2011 Aug;25(8):873-84.

18 Rittié L, Fisher GJ. Natural and sun-induced aging of human skin. Cold Spring Harb Perspect Med. 2015 Jan;5(1):a015370.

19 Farage MA, Miller KW, Elsner P, Maibach HI. Characteristics of the aging skin. Adv Wound Care (New Rochelle). 2013 Feb;2(1):5-10.

20 Zouboulis CC, Makrantonaki E. Clinical aspects and molecular diagnostics of skin aging. Clin Dermatol. 2011 Jan-Feb;29(1):3-14.

21 Mokos ZB, Ćurković D, Kostović K, Čeović R. Facial changes in the mature patient. Clin Dermatol. 2018 Mar - Apr;36(2):152-8.
22 Thiele JJ, Schroeter C, Hsieh SN, Podda M, Packer L. The antioxidant network of the stratum corneum. Curr Probl Dermatol. 2001;29: $26-42$.

23 Oresajo C, Pillai S, Yatskayer M, Puccetti G, McDaniel D. Antioxidants and Skin aging: a review. Cosmetic Dermatol. 2009;22:563-8.

24 Starfield B, Hyde J, Gérvas J, Heath I. The concept of prevention: a good idea gone astray? J Epidemiol Community Health. 2008 Jul; 62(7):580-3.

25 Bickers DR, Athar M. Oxidative stress in the pathogenesis of skin disease. J Invest Dermatol. 2006 Dec;126(12):2565-75.

26 Trüeb RM. 10th World Congress of Hair Research. Global view on extrinsic and intrinsic factors affecting hair fiber quality of life. Kyoto, Japan: Oral communication; 2017.

27 Trüeb RM, Tobin DH, editors. Aging Hair. Berlin/Heidelberg: Springer; 2010.

28 Papageorgiou N, Carpeter E, Tobin DJ. Human hair follicle melanocytes as a proxy cell type in neurodegenerative research. In: Trüeb RM, Tobin DH, editors. Aging Hair. Berlin/ Heidelberg: Springer; 2010. p. 101.

29 Trüeb RM, Dias MF. A comment on trichoscopy. Int J Trichology. 2018 Jul-Aug; 10(4): 147-9.

30 Trüeb RM, Rezende HD, Diaz MF. Dynamic Trichoscopy. JAMA Dermatol. 2018 Aug; 154(8):877-8.

31 Kaufman KD. Androgen metabolism as it affects hair growth in androgenetic alopecia. Dermatol Clin. 1996 Oct;14(4):697-711.

32 Randall VA, Thornton MJ, Messenger AG. Cultured dermal papilla cells from androgendependent human hair follicles (e.g. beard) contain more androgen receptors than those from non-balding areas of scalp. J Endocrinol. 1992 Apr;133(1):141-7. 
33 Itami S, Kurata S, Takayasu S. Androgen induction of follicular epithelial cell growth is mediated via insulin-like growth factor-I from dermal papilla cells. Biochem Biophys Res Commun. 1995 Jul;212(3):988-94.

34 Trüeb RM. Further clinical evidence for the effect of IGF-1 on hair growth and alopecia. Skin Appendage Disord. 2018 Apr;4(2):90-5.

35 Naito A, Midorikawa T, Yoshino T, Ohdera M. Lipid peroxides induce early onset of catagen phase in murine hair cycles. Int $\mathrm{J} \mathrm{Mol}$ Med. 2008 Dec;22(6):725-9.

36 Bahta AW, Farjo N, Farjo B, Philpott MP. Premature senescence of balding dermal papilla cells in vitro is associated with p16(INK4a) expression. J Invest Dermatol. 2008 May; 128(5):1088-94.

37 Trüeb RM. 10th World Congress of Hair Research. Medical and cosmetic treatments for aging male and female hair. Kyoto, Japan: Oral communication; 2017.
38 Schwartz JR, Henry JP, Kerr KM, Flagler MJ, Page SH, Redman-Furey N. Incubatory environment of the scalp impacts pre-emergent hair to affect post-emergent hair cuticle integrity. J Cosmet Dermatol. 2018 Feb;17(1):10511.

39 Trüeb RM, Henry JP, Davis MG, Schwartz JR. Scalp condition impacts hair growth and retention via oxidative stress. Int J Trichology. 2018 Nov-Dec;10(6):262-70.

40 Trüeb RM. Association between smoking and hair loss: another opportunity for health education against smoking? Dermatology. 2003; 206(3):189-91.

41 Price VH, Sawaya ME, Headington JT. Histology and hormonal activity in senescent thinning in men. Presentation at SID, Annual Meeting; Washington, DC. 2001.

42 Trüeb RM. Minoxidil for endocrine therapyinduced alopecia in women with breast cancer - Saint Agatha's blessing? JAMA Dermatol. 2018 Jun;154(6):656-8.

43 Trüeb RM. Dermocosmetic aspects of hair and scalp. J Investig Dermatol Symp Proc. 2005 Dec;10(3):289-92.
44 Trüeb RM. Shampoos: ingredients, efficacy and adverse effects. J Dtsch Dermatol Ges. 2007 May;5(5):356-65.

45 Gavazzoni Dias MF. Hair cosmetics: an overview. Int J Trichology. 2015 Jan-Mar;7(1):215.

46 Gavazzoni Dias MF, de Almeida AM, Cecato $\mathrm{PM}$, Adriano AR, Pichler J. The shampoo $\mathrm{pH}$ can affect the hair: myth or reality? Int J Trichology. 2014 Jul;6(3):95-9.

47 Marsh JM, Davis MG, Lucas RL, Reilman R, Styczynski PB, Li C, et al. Preserving fibre health: reducing oxidative stress throughout the life of the hair fibre. Int J Cosmet Sci. 2015 Dec;37 Suppl 2:16-24.

48 Davis MG, Thomas JH, van de Velde S, Boissy Y, Dawson TL Jr, Iveson R, et al. A novel cosmetic approach to treat thinning hair. $\mathrm{Br} J$ Dermatol. 2011 Dec;165 Suppl 3:24-30.

49 Ahluwalia J, Fabi SG. The psychological and aesthetic impact of age-related hair changes in females. J Cosmet Dermatol. 2019 Aug;18(4): $1161-9$. 